\title{
Charging Control Strategy to reduce the remaining capacity of electric buses
}

\author{
Yachao Hu ${ }^{\mathrm{a}}$, Xia Chen ${ }^{\mathrm{b}}$, Aiyu Wang ${ }^{\mathrm{c}}$, Wenguan Jia, Cancan Kong, Fengyin \\ Zhao, Xingcheng Nie, Zhengtong Lu \\ Shandong University of Science and Technology, Qingdao 266590, China \\ ayachao0416@163.com, bsdkdcx@126.com, '1032551550@qq.com
}

Keywords: Control policy, Bus Charging Station, Battery.

\begin{abstract}
In order to solve the electric buses remaining excess capacity at the end of work and the economic operation problems of the power grid, the too large load and the higher harmonic content during the noon to complement the power collectively. In this paper the electric bus charging station is the study object, analyzes the negative effect on the gird due to the too much electric buses remaining battery, present a charging control policy to reduce the remaining battery of electric buses depending on the TOU (time of use)and the running characteristics of bus charging stations. The simulation based on practical cases indicated that the policy can be a good way to decrease the remaining battery at the end of work, reduce the grid maximum load and cut the cost of the bus charging station.
\end{abstract}

\section{Introduction}

With the increasing of global energy crisis, oil and other fossil fuels dwindling, the global climate and environment becoming worse. Reduce the dependence on oil, natural gas and other fossil fuels has become an urgent problem in the world, this problem can be solved by developing electric buses, while reducing the pressure of urban traffic [1]. However, large-scale development of electric buses will bring lots of charging stations into the gird to increase the burden on the gird and affect the power quality. So the study of electric bus charging control policy is particularly important.

At present, research on the electric vehicle charging control policy focuses on private cars [2], engineering vehicles ${ }^{[4]}$. But private cars and engineering vehicles do not have the indefinite time and the places are not uniform, it is difficult to control accurately. The charging time of electric bus is fixed and the location is centralized, it has a strong law. So it has a strong feasibility to study electric bus charging control policy.

In this paper, analyzes the negative effect on the gird due to the too much remaining battery of electric buses,present a charging control policy to reduce the remaining battery of electric buses depending on the adaptive mutation particle swarm optimization and the running characteristics of bus charging stations. The validity of this method is testified through simulation examples.

\section{Impact of electric buses remaining capacity on the grid}

Bus stations give the supplement for battery power during the daytime 11:00-13:30, all the buses charging at the end of work during night. Charge monitoring platform show too much buses remaining battery, the too much battery is an extra part, it is from the supplement for battery during 11:00-13:30, which means there are too much supplement during that time. The too much battery will make the peak load condition of the gird worse during noon, higher harmonic content and affect the power quality [3], it also has a great of waste and decrease the efficiency.

\section{Bus charging stations charging load model}

Forecast the daily load curve based on GRNN [5] General Regression Neural Network, the interval of daily load curve is $0.5 \mathrm{~h}$, the amount of data sampling of the curve can be 48 , Charging load model is: 


$$
W_{x}= \begin{cases}\sum_{i=1}^{N-P} W_{i, x} & , 22-27 \\ \sum_{i=1}^{N} W_{i, x}+\sum_{j=1}^{P} W_{j, x} & , 45-10\end{cases}
$$

Wherein: $W_{x}$ is the $x$ th time interval of charging load in every day, $x=[1,48] ; W_{i, x}$ is the charging load of the $x$ th bus during the x time interval; A total of $N$ electric buses; Each electric buses electricity equal to a set of battery charge, the battery number is $j$,a total of $P$ set of battery; 22-27 is the bus supplementary charge time 11:00-13:30; 45-10 is centralized charging time in the night 22:30-05:00.

Formula (1) take the initial SOC value of the bus battery into consideration, after a day's work complete the value of battery $S O C_{d o}$ is bigger than $S O C_{p r}$ which is required for protecting the battery. The ideal state of battery is $S O C_{d o}=S O C_{p r}$, the model is:

$$
W_{x}^{\prime}=\left\{\begin{array}{l}
\sum_{i=1}^{N-P} W_{i, x}^{\prime}<\sum_{i=1}^{N-P} W_{i, x}, 22-27 \\
\left(\sum_{i=1}^{N} W_{i, x}^{\prime}+\sum_{j=1}^{P} W_{j, x}^{\prime}\right)>\left(\sum_{i=1}^{N} W_{i, x}+\sum_{j=1}^{P} W_{j, x}\right), 45-10
\end{array}\right.
$$

After decreased the remaining SOC value, the total load is decreased during the supplementary charging time 22-27 from formula(2), the load of centralized charging time 45-10 is increased, which means the too much battery is moved into night $45-10$ from daytime $22-27$. Meet the requirements of grid scheduling.

\section{Economic Benefit Analysis}

There will be significant economic benefits to reduce electric buses remaining battery for electric bus station.

The ways for estimating SOC of electric buses are mostly improved ampere-hour integral method ${ }^{[9]}$, but the error of improved ampere-hour integral method will be continue to accumulate with the passage of time. In this paper, Kalman filtering method is used to estimate the value of electric bus battery SOC so that continue accumulating errors can be avoided.

Equation of state:

$$
X_{K+1}=A_{K} X_{K}+B_{K} U_{K}=\mathrm{f}\left(X_{K}, U_{K}\right)+W_{K}
$$

Observation equation:

$$
Y_{K+1}=C_{K} X_{K}+V_{K}=g\left(X_{K}, U_{K}\right)+V_{K}
$$

Wherein: $U_{K}$ is system input vector contains the current, temperature and remaining capacity of battery, $Y_{K}$ is system output vector contains the working voltage of battery, $X_{K}$ is the SOC value of the battery.

Each electric buses added superfluous electricity tariff $\mathrm{f}$ is:

$$
f=\sum_{t=x}^{\frac{\left(S_{s t}-S_{p r}\right)}{P \cdot \Delta t}} P \cdot \Delta t \cdot c_{x}-\sum_{t=y}^{\frac{\left(S_{s t}-S_{p r}\right)}{P \cdot \Delta t}} P \cdot \Delta t \cdot c_{y}
$$

Formula (5): $S_{s t}$ is the remaining power at the end of the whole day work; $S_{p r}$ is the power for the battery protection; $f$ is charger charging power; $\Delta t$ is the length of time $c_{x}$ and $c_{y}$ are both day and night tariff respectively, as shown in Tab1.

Tab.1 Time of use power price

\begin{tabular}{cc}
\hline Time Period & $\begin{array}{c}\text { Grid TOU } \\
(\text { Yuan/(kw·h) })\end{array}$ \\
\hline $00: 00-08: 00$ & 0.332 \\
$08: 00-12: 00$ & 0.796 \\
$12: 00-14: 30$ & 0.667 \\
21:00-24:00 & 0.633 \\
\hline
\end{tabular}




\section{Electric bus charging control policy}

Methods for reducing the remaining battery electric of buses. Reference [10] specifies the test methods for electric vehicles energy loss rate and mileage. According to the schedule of electric bus station and the statistical analysis of electric buses mileage, forecast the needed electricity $S_{n e}$ of electric bus to complete the remaining in the afternoon. Which means that supplementary charge stage 22-27 is just provide the necessary electricity in the afternoon.

Charge control policy. In order to avoid the problem of higher peak load, stabilize the load fluctuations and lower the peak-valley difference, in this paper and peak-valley difference are objective function.

The objective function is the minimum load fluctuations:

$$
F=\min \left\{\sum_{t=1}^{48}\left[\left(\sum_{i} P_{i, t}+P_{\text {load }, t}-u\right)^{2}\right]\right\} / 48
$$

The objective function is the minimum peak-valley difference:

$$
G=\min \left[\max \left(P_{\text {load }, t}^{\prime}\right)-\min \left(P_{\text {load }, t}^{\prime}\right)\right]
$$

The constraints of electric bus charging station control policy are the constraint of charging time, the SOC value of bus battery and the total charging power of charging station.

The constraint of charging time:

Supplementary charge time: $22 \leq t \leq 27$

Centralized charging time: $45 \leq t \leq 10$

The constraint of the total charging power:

$$
P_{z, t} \leq P_{\text {set }}
$$

$P_{\text {set }}$ is the limited value of grid

The constraint of bus SOC value:

$$
S O C_{i, \min } \leq S O C_{i, t} \leq S O C_{i, \max }
$$

Formula (14): $S O C_{i \min }$ and $S O C_{i \max }$ are the minimum and maximum values of every electric bus battery respectively.

Adaptive mutation particle swarm optimization. Particle swarm optimization is vulnerable to premature convergence, PSO algorithm is optimized in this paper so that the problem can be avoided.

Let $f_{i}$ be the fitness of the particle, The average fitness of all the $v$ particles can be get from formula (12),the normalization $f$ of PSO can be determined from formula (13), formula(14) give the variance of the population's fitness.

$$
\begin{aligned}
& f_{\text {avg }}=\frac{1}{v} \sum_{i=1}^{v} f_{i} \\
& f=\left\{\begin{array}{c}
\max \left\{\left|f_{i}-f_{\text {avg }}\right|\right\}, \quad \max \left\{\left|f_{i}-f_{\text {avg }}\right|\right\}>1 \\
1, \quad \text { other }
\end{array}\right. \\
& \sigma^{2}=\sum_{i=1}^{v}\left(\frac{f_{i}-f_{\text {avg }}}{f}\right)^{2}
\end{aligned}
$$

There will be variation of best if it meets conditions of variation, the expressions of $p_{m}$ and $p_{m}$ are:

$$
p_{m}=\left\{\begin{array}{l}
u, \sigma^{2}<\sigma_{d}^{2}, f(\text { gbest })>f_{d} \\
0, \text { other }
\end{array}\right.
$$

Wherein: $u$ can take any value between $[0.1,0.3] ; f_{d}$ is set to be the theoretical optimal value. Take random perturbation method for the variation of best, set best to the value of $I$ the dimension of best, $\eta$ is subject to Gaussian distribution.

$$
\text { gbest }_{i}=\text { gbest }_{i}(1+0.5 \eta)
$$

Update particle position formula: 
$v_{I d}^{k+1}=w v_{I d}^{k}+c_{1}$ rand $_{1}^{k}\left(\right.$ pbest $\left._{I d}^{k}-x_{I d}^{k}\right)+c_{2}$ rand $_{2}^{k}\left(\right.$ gbest $\left._{I d}^{k}-x_{I d}^{k}\right)$

Update particle velocity formula:

$$
x_{I d}^{k+1}=x_{I d}^{k}+v_{I d}^{k+1}
$$

\section{Algorithm Process}

According to the schedule of electric bus station and the statistical analysis of electric buses mileage, forecast the needed electricity $S_{n e}$.

Adjust charging power of bus station during supplementary charge time, reduce the remaining battery of electric bus;

Optimize the centralized charging time based on the Adaptive Mutation Particle Swarm Optimization;

Initialize the position and velocity of particles;

Modify the position of the particle according to the constraint formula (8)-(11);

Calculate the fitness of particle swarm, recorded the best position of individual particles and groups;

Update velocity and position of particle according to formula (17) and (18);

Calculate the variance $\sigma^{2}, f$ and mutation probability pm according to formula (12) and (15);

Generates a random number $r \in[0,1]$, if $r<p_{m}$, perform mutation operations according to formula (16), check the position of particles whether beyond the limit, if beyond the limit repeat step 5);

Calculate the fitness of particle swarm, update and record the particle best position and the groups;

The calculation will be stopped if reached the end condition, otherwise return to step 7).

\section{Example}

Select the gird daily load curve of an area as the original load curve for the study, as the curve shown in Fig.1. Study load changes after using optimal control strategy based on the biggest electric bus charging station in the area. The maximum capacity of the battery in the station is $55 \mathrm{MWH}$, the maximum charging power is $12 \mathrm{MW}$.

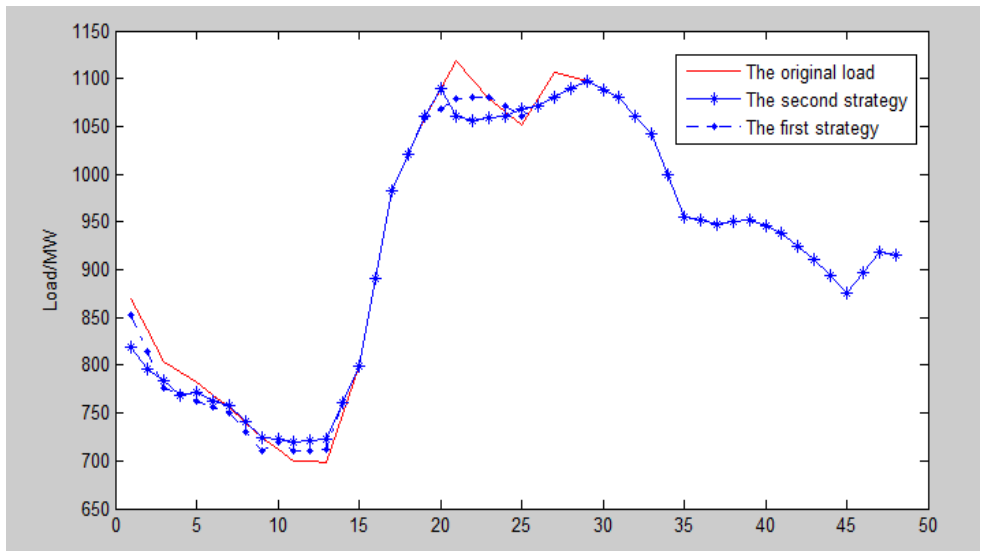

Fig.1 The optimized load curve

The first strategy in Fig.1 is the results of optimization of adaptive mutation particle. The second strategy is the combination of reduce the remaining capacity of electric buses and optimization of adaptive mutation particle. From the curve in Fig.1, it can be seen the second curve during supplementary charge stage 22-27 and Centralized charging phase 45-10 is much lower than the original daily load curve after the optimization. Based on the simulation result, the peak-valley difference can be reduced and cut operating costs of electric bus charging station. 


\section{Summary}

This paper analyzes the negative effect on the gird due to the too much remaining battery of electric buses, present a charging control policy to reduce the remaining battery of electric buses depending on the adaptive mutation particle swarm optimization. The result shows that the control policy can move the too much battery into night 45-10 from daytime 22-27. Meet the requirements of grid scheduling, realize peak clipping and valley filling and bring great economy profit.

\section{Acknowledgements}

This work was financially supported by the Shandong University of Science and Technology Postgraduate Innovation Fund Projects YC: 140335.

\section{References}

[1] Cao Bing-gang. Current progress of electric vehicle development in China [J].Journal of Xi'an Jiaotong University, 2007, 41(1): p.114-118.

[2] Gao Ciwei, Zhang Liang. A survey of influence of electrics vehicle charging on power grid [J]. Power System Technology, 2011, 35 (2): p.127-131(in Chinese).

[3] Bao Guannan, Lu Chao, Yuan Zhichang, et al. Load shift real-time optimization strategy of battery energy storage system based on dynamic programming[J].Automation of Electric Power Systems.2011,35(12): p.11-16.

[4] Zhao Junhua, Wen Fushuan, Yang Aimin, et al. Impacts of electric vehicles on power systems as well as the associated dis patching and control problem[J], Automation of Electric Power Systems, 2011,35(14): p.2-10(in Chinese).

[5] Wang Zhiyong, Guo Chuangxin, Cao yijia, a method for short term load forecasting integrating fuzzy-rough set with artificial neural network [J]. Processing of the CSEE, 2005, 25(19): p.7-11.

[6] TIAN Wen-qi, HE Jinghan, JIANG Jiu-chun, et al. Multi-Objective Optimization of Charging Dispatching for Electric Vehicles Battery Swapping Station Based on Adaptive Mutation Particle Swarm Optimization[J]. Power System Technology, 2012, 40(21): p.25-29.

[7] YU Zhisheng. Automobile theory [M]. Beijing: Machinery Industry Press, 2009

[8] A. Maitra, et al. integrating plug-in electric vehicles with the distribution system. $20^{\text {th }}$ International Conference and Exhibition on Electricity Distribution (CIRED 2009), 8-11 June 2009, Steven age, UK, 2009: p.1029-1034.

[9] HUA Zhou-fa, LI Jing. Summary of methods for state of charge estimation of EV power batteries [J]. Chinese journal of Power Source, 2013, 37(9): p.1686-1689.

[10] China National Standardization Management Committee. GB/T 18386-2005 《Electrics-Energy consumption and range-Test procedures 》[S]. China Zhijian Publishing House. 2005. 